\title{
2 Von der Bedeutungslosigkeit des Praxiswartezimmers
}

\author{
Christian Gondek, Shabnam Fahimi-Weber und David Matusiewicz
}

Es gibt Industrien, die die digitale Transformation erfolgreich gemeistert haben. In denen Kunden im Mittelpunkt stehen, die digitale Symbiose zwischen persönlichem Service und digitaler Bedienung zu einem perfekten Kundenerlebnis beiträgt. In denen die Wartezeit der einer Dienstleistung verbrauchenden Konsumenten wertgeschätzt wird und quasi nicht mehr existent ist. Die Medizinbranche gehört mit Sicherheit nicht dazu.

Wie kann dies in einer der historisch betrachtet fortschrittlichsten Branchen sein, und wie ist es so weit gekommen? Bereits seit Jahren gilt als akademisches industrieübergreifendes Grundwissen eine Kausalität zwischen der Zugänglichkeit einer Dienstleistung und der Zufriedenheit, Loyalität und Wiederinanspruchnahme eben dieser (Dixon et al. 2010). Nicht zuletzt dadurch wird die Unvereinbarkeit des sich hinter einem Wartezimmer verschanzendem unnahbaren Ärzteapparates mit der Zufriedenheit der Patienten offensichtlich. Denn vor allem in der Medizinbranche gilt, je geringer die Wartezeit der Patienten, desto zufriedener und einfacher zu behandeln gilt der Patient. Als direkte Auswirkung wird auch die Behandlung für Ärzte entspannter, zielgerichteter und effizienter (Meyer et al. 2016).

Viele Ärzte argumentieren sicherlich mit der Qualität der Behandlung, die unabhängig der Wartezeit immer im Vordergrund stehen sollte. Hierbei gilt zu beachten, dass die Zufriedenheit ihrer Patienten auf einer Gleichung aus reibungslosen Abläufen, Wartezeiten und gleichermaßen der Qualität Ihrer Behandlung basiert (Meyer at al. 2016). Und machen Sie sich nichts vor: In den nächsten Jahren wird die Digitalisierung vor kaum einer Aufgabe, die Sie heute noch als selbstverständlich erachten, haltmachen. Diagnosen: werden von Maschinen übernommen. Behandlungspläne: 
erfolgen automatisch. Historische Krankheitsentwicklungen: werden ausgelesen, analysiert und grafisch für Sie aufbereitet.

Die essenziellen Interaktionsteilnehmer einer Ärzte-Patienten-Beziehung werden jedoch weiterhin Bestand haben, menschlicher Natur sein und menschliche Bedürfnisse haben. Sie werden gereizt, verängstigt oder besorgt sein. Sie werden gesund, krank, gut oder schlecht gelaunt und mit viel oder wenig Zeit sein. Sie werden also im wahrsten Sinne des Wortes menschlich und somit individuell bleiben und eine individuelle Behandlung benötigen.

Es gibt also auch gute Nachrichten für alle Ärzte: Die digitale Transformation wird niemals zu einer rein digitalen Lösung führen können. Viel wichtiger gilt es, eine ausgewogene Balance zwischen digitalen Lösungen und einer menschlichen, fürsorglichen und vertrauenswürdigen Behandlung zu erzielen. 83\% der deutschen Verbraucher möchten lieber mit Menschen agieren, und 77\% erwarten persönliche Gespräche und Beratung. Dem gegenüber stehen 61\% der deutschen, die sich ein höheres Servicetempo wünschen (Accenture 2016a).

Lassen Sie uns offen sprechen: Es wird auch in den nächsten Jahren ältere Patienten geben, die mit ihrem handgeschriebenem Kalender in Ihrer Praxis einen Tag nach dem anderem aufschlagen und einen Tag nach dem anderen in einem gefühlt zehnminütigen Gespräch mit Ihrem Praxispersonal überprüfen. Demgegenüber steht die Zukunft - die Millennials. Und die wollen immer mehr digitale Erlebnisse von Ihnen an jedem möglichen Touchpoint ihres Behandlungserlebnisses (Accenture 2016b).

Bereits heute hat die Digitalisierung - teilweise ungewollt - Einzug in das Verhalten vieler Patienten genommen oder haben Sie etwa noch nicht von der Cyberchondrie gehört? Das passiert, wenn Ihre Patienten einem ureigenen Bedürfnis Rechnung tragen, der Selbstdiagnose Ihrer eigenen Symptome, und sich dadurch in eine regelrechte Angst vor Krankheiten hineinsteigern. Schlimmer noch wird es, wenn diese Patienten mit der Selbstbehandlung beginnen, sodass Sie hinterher nur noch die entstandenen Schäden von Übermedikation und falschen Diagnosen begradigen dürfen.

Also, wenn die Digitalisierung der Medizinbranche unaufhaltsam ist, wie sieht dann ein gesteuertes und gewolltes Szenario der Zukunft aus? Lassen Sie es uns zusammen vorstellen: Ihre Patienten geben ihr wahrgenommenes Symptom in ein digitales Medium ein und werden zielgerichtet zu dem passenden Spezialisten weitergeleitet. Ein Termin erfolgt zeitnah und individuell für jeden Patienten. Individuell bedeutet hierbei in einem perfekten Zusammenspiel aus den zeitlichen Wünschen des Patienten und der wirtschaftlich notwendigen Interessen der Praxis. Gleichermaßen wird es für Ihre Patienten keinen Unterschied mehr machen, Ihre Dienstleistung mit irgendeiner anderen zu vergleichen und zu bewerten. Somit ändern sich die Erwartungen Ihrer Praxis gegenüber und werden eher wie gegenüber jedem anderen wirtschaftlichen Betrieb.

Wartezeiten sind dann nicht mehr existent, denn Ihr Patient hat eine transparente Übersicht über den kompletten Behandlungszeitraum. Das heißt im Klartext, dass Wartezimmer bedeutungslos geworden sind. Es wird keine wartenden, kranken und gereizten Patienten mehr geben. Auch Nachfragen zu Behandlungsterminen haben sich erübrigt. Damit schaffen Sie eine optimale Symbiose zwischen der Effizienz und Effektivität Ihres Praxispersonals, das sich heute noch mit teilweise 70 Anrufen pro Tag und dutzenden persönlichen Nachfragen zur Wartezeit herumschlagen muss. 
Als Ergebnis genießen Sie ein vertrauensvolles Ärzte-Patienten-Verhältnis mit zufriedenen und loyalen Patienten. Oder Sie haben sich der Digitalisierung enthalten. Dann sind Sie in einigen Jahren entweder im Ruhestand oder ohne Patienten.

\section{Praxisexkurs - Patientenfokussiertes Terminmanagement}

Das Prinzip eines Essener Start-ups basiert auf einem patientenfokussierten Terminmanagement. Ausgehend von der Frage: Was wünschen sich eigentlich die Patienten?

- Terminvereinbarung unabhängig von Ort und Zeit

n weniger zeitintensive Telefonie um einen Termin zu bekommen

- Freiheit bei der Terminwahl

- schnelle, aber qualitativ gute medizinische Hilfeleistung

- weniger Wartezeit in der Praxis

- Patientenströme werden nach den Bedürfnissen der Praxisinhaber aktiv geplant. Das System vergibt automatisch einen schnellen Termin an Patienten, denen der Arzt einen Vorrang gewähren möchte. Die Anzahl der Wiederholer im Quartal nimmt ab.

Dabei warten die Patienten im Durchschnitt 6 Stunden und 48 Minuten jährlich in den Wartezimmern einer Arztpraxis. Warten macht dabei unzufrieden. Das häufige Nachfragen „Wann bin ich dran?" und die Unruhe in einem vollen Wartezimmer tragen häufig zu einer anstrengenden Arbeitsstimmung bei den Mitarbeitern und Ärzten bei. Nicht zu wissen, wie lange man warten muss, verlängert die subjektiv empfundene Wartezeit. Die Zufriedenheit kann dabei durch erhöhte Transparenz der Wartedauer geschaffen werden. Transparenz über Wartezeit reduziert den kollektiven Stress und erhöht die Zufriedenheit bei Mitarbeitern und Patienten. $3 \ldots 2 \ldots 1 \ldots$ - aufstehen und direkt ins Wartezimmer durchmarschieren.

\section{Wie funktioniert das Ganze?}

Zunächst wird das Symptom eingegeben bspw. Kopfschmerzen. Das Programm bietet Termine bei Ärzten verschiedener Fachrichtungen an, die zur Behandlung infrage kommen; z.B. Neurologen, Allgemeinmediziner, Orthopäden oder Internisten. In einem elektronischen Wartezimmer können Patienten einsehen, wie lange es noch bis zu ihrem Behandlungsbeginn dauert. Der Patient kann diese Zeit nach seinen Bedürfnissen nutzen. Netter Nebeneffekt: für die Praxis ist eine Umsatzsteigerung durch Erhöhung der „Scheinzahl“ bei gleichbleibendem Personalschlüssel möglich (in einer Referenzpraxis im Testpilot in Essen um 18\%).

\section{Vorteile auf einen Blick - dubidoc 2017}

- Reduktion der Verwaltungsvorgänge in der Praxis und damit freie Ressourcen bei medizinischem Personal für die Patientenversorgung.

- Die sofortige Verwertung der abgesagten Termine führt dazu, dass mehr freie Termine in den Pool einfließen. 


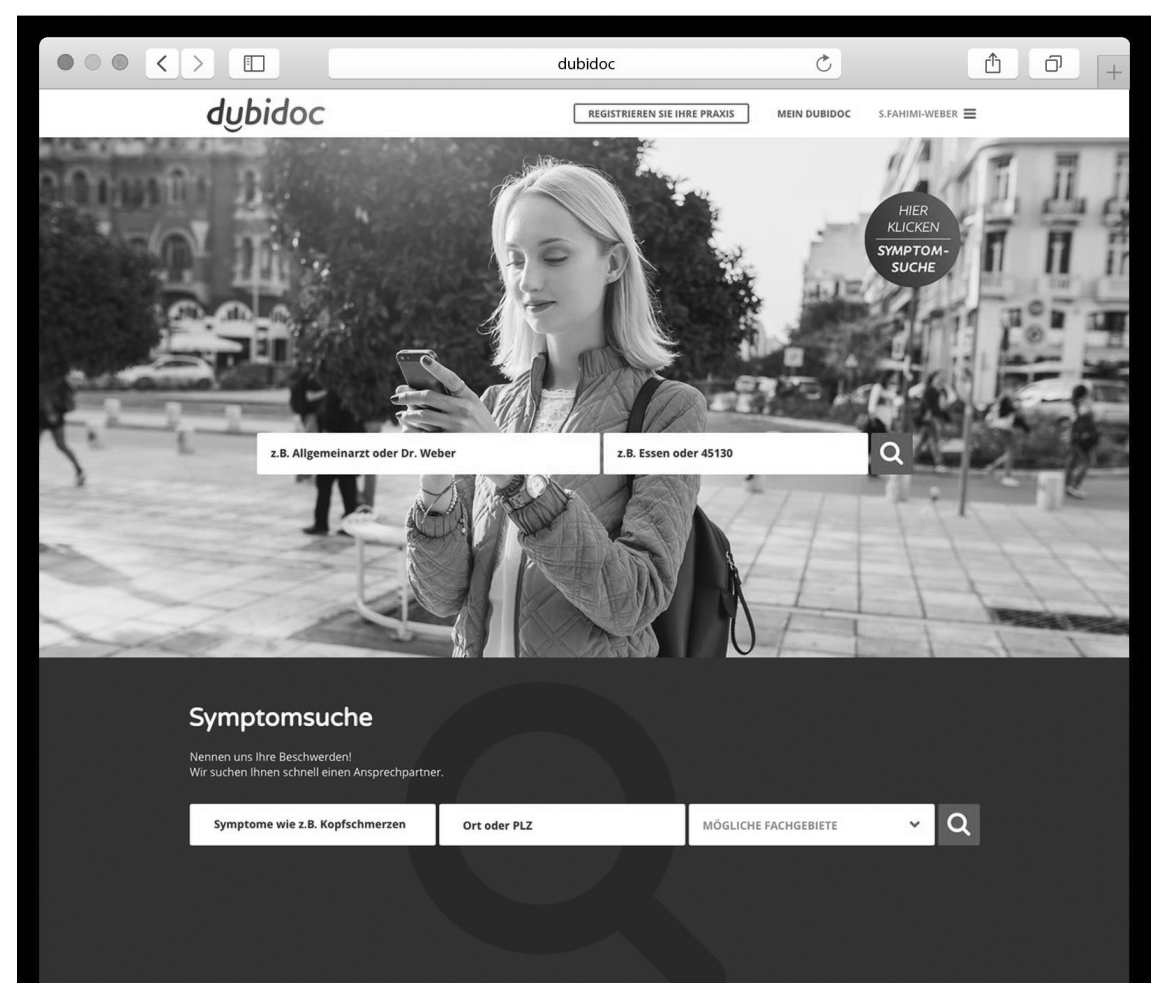

Abb. 1 dubidoc Symptomsuche

- Die Symptomsuche (s. Abb. 1) erlaubt den Patienten, auf größere Auswahl an Behandlermöglichkeiten, die zur Behandlung seiner Erkrankung infrage kommen und möglicherweise einen früheren Termin haben, zurückzugreifen.

- Transparenz über die Wartezeit schafft freie Ressourcen bei den Patienten und hat Einfluss auf Ihr Zeitmanagement.

Die Autoren machen allerdings auch darauf aufmerksam, dass es sicherlich eine kritische Diskussion ist, ob die Zielzahl der Wartezeit = 0 (Tage bzw. Stunden bzw. Minuten) sein soll. Manchmal kann auch eine angemessene Wartezeit hilfreich sein, um nicht überflüssig Ressourcen im Gesundheitswesen in Anspruch zu nehmen oder die Zeit zur Reflexion zwischen den Arztterminen zu haben.

\section{Literatur}

Accenture (2016a) Digitaler Bruch in der Kundenbeziehung, URL: https://www.accenture.com/de-de/insightdigital-disconnect-customer-engagement (abgerufen am 28.07.2017)

Accenture (2016b) Is your channel strategy pushing your most profitable customers away, URL: https:// booksmovie.org/similar-pdf-ustomers-are-the.html (abgerufen am 28.07.2017) 


\section{Entrepreneure und Start-ups}

Dixon M, Freeman K, Toman N (2010) Stop trying to delight your customers. Harvard Business Review 88.7/8, $116-122$

dubidoc (2017) dubidoc Imagefilm, URL: https://www.youtube.com/watch?v=DToDY2-2ZOs\&app=desktop (abgerufen am 28.07.2017)

Meyer C, Bartsch D K, Fendrich V (2016) Korrelation von Wartezeit und Patientenzufriedenheit in einer chirurgischen Poliklinik. Deutsche Gesellschaft für Chirurgie. 133. Kongress der Deutschen Gesellschaft für Chirurgie. Berlin, 26.-29.04.2016. Düsseldorf: German Medical Science GMS Publishing House; 2016. Doc16dgch127 